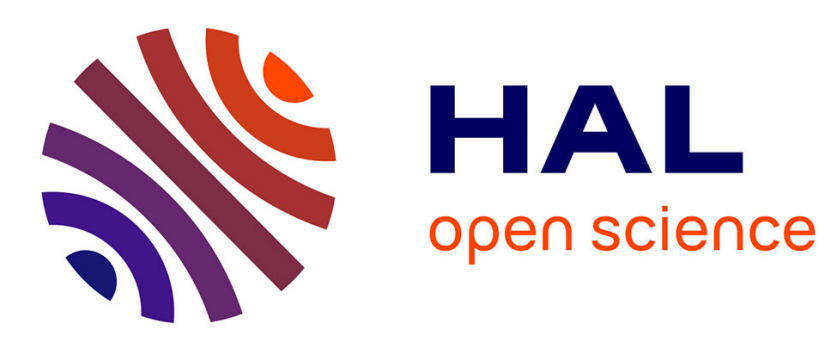

\title{
Closed-looping a P300 BCI using the ErrP
}

\author{
Sandra Rousseau, Christian Jutten, Marco Congedo
}

\section{To cite this version:}

Sandra Rousseau, Christian Jutten, Marco Congedo. Closed-looping a P300 BCI using the ErrP. NCTA 2012 - 5th International Conference on Neural Computation Theory and Applications, Oct 2012, Barcelone, Spain. pp.1. hal-00741236

\section{HAL Id: hal-00741236 https://hal.science/hal-00741236}

Submitted on 12 Oct 2012

HAL is a multi-disciplinary open access archive for the deposit and dissemination of scientific research documents, whether they are published or not. The documents may come from teaching and research institutions in France or abroad, or from public or private research centers.
L'archive ouverte pluridisciplinaire HAL, est destinée au dépôt et à la diffusion de documents scientifiques de niveau recherche, publiés ou non, émanant des établissements d'enseignement et de recherche français ou étrangers, des laboratoires publics ou privés. 


\title{
Closed-looping a P300 BCI using the ErrP
}

\author{
Rousseau Sandra ${ }^{1}$, Jutten Christian ${ }^{1}$ and Congedo Marco ${ }^{1}$ \\ ${ }^{1}$ Gipsa-lab-DIS, 11 rue des mathématiques Domaine Universitaire, 38400 Saint-Martin d'Héres, France \\ sandra.rousseau@gipsa-lab.grenoble-inp.fr
}

\begin{abstract}
Keywords: $\quad$ BCI, Error-related potential, closed-loop.
Abstract: $\quad$ The error-related potential is an event-related potential that gives information on the quality (error or correct) of what a subject observes. In this paper we try to integrate it in a P300 BCI system in order to introduce a closed-loop in this system and thus to improve its accuracy. We propose and compare different strategies of integration and discuss on their possible improvements depending on our system characteristics. We get a mean improvement of $10 \%$ of our system when using the error-related potential to correct errors.
\end{abstract}

\section{Introduction}

$\mathrm{BCI}$ are systems that aim at decoding brain signals and convert them into orders for driving an external device. One very well known BCI is the P300 speller which allows a subject to spell words using a virtual keyboard. Actual BCIs performances need to be improved in order for them to be more easily usable. One way of improving this accuracy is to include a control loop based on error detection which can be performed with the error-related potential. Errorrelated potentials (ErrP) are evoked potentials which are generated when a subject makes or observes an error (Falkenstein et al., 1991),(Gehring et al., 1993). It is time-locked to the observation of the error and is mostly characterized by a negative deflection (Ne) (Gentsch et al., 2009), followed by a large positivity (Pe) (Steinhauser and Kiesel, 2011). Single-trial detection of this potential is now the subject of many studies. As any ERP, the signal to noise ratio of the ErrP is very low and it is hard to see it on a single trial basis. However most studies reached in average a rate of $70 \%$ of good detection for error trials and a rate of $80 \%$ of good detection for correct trials (Ferrez and Millán, 2005),(Chavarriaga et al., 2007),(Bollon et al., 2009). In this paper we study the possible integration of the ErrP in a P300 BCI as an error correction system. Indeed, when the BCI makes an error, an ErrP appears in the subject's EEG. If one can detect this ErrP then it could be used as a control loop to correct errors made from the BCI. Some authors already studied thie issue in two to four clas BCIs (Ferrez and Millán, 2007),(Ferrez and Millán, 2008) or in multiclass BCIs (Dal Seno et al., 2010).
Here we present different kind of strategies that can be used to correct BCI errors in a P300 speller and what kind of improvement can be achieved. First we study the characteristics of different P300 datasets in order to see the repartition of errors and corrects probabilities. Then, using these observations, we propose different stragegies for ErrP integration and test them on our datasets according to different performances of our system. Finally we discuss on their different advantages and disadvantages.

\section{Studying a P300 speller}

In order to determine what kind of strategy could be used to integrate ErrPs in a P300 speller we study the characteristics of a P300 system.

\subsection{Datasets}

For this study we used two datasets available online from BCI competitions (Blankertz et al., 2004) (http://www.bbci.de). The two data sets come from the same paradigm (Farwell and Donchin, 1988). The user was presented with a 6 by 6 matrix of characters . The user's task was to focus attention on characters that were prescribed by the investigator (one character at a time). For each character, all rows and columns of this matrix were successively and randomly intensified (leading to 12 intensifications). This was repeated 15 times (leading to $15 \times 12=180$ intensifications for each character). For each character, user display was as follows: the matrix was blank for $2.5 \mathrm{~s}$. Then each row and column was randomly intensified for $100 \mathrm{~ms}$. 
After intensification of a row/column, the matrix was blank for $75 \mathrm{~ms}$. Then a $2.5 \mathrm{~s}$ period of rest was allowed during which the matrix was blank. Subjects were asked to spell predefined words separated into different sessions. For the first dataset, signals were collected from two subjects in five sessions each.For the second dataset signals were collected from one subject in three sessions. All data were digitized at $240 \mathrm{~Hz}$.

\subsection{Preprocessing and classification}

The same method was used for every subject. Data were bandpass filtered between $0.2-20 \mathrm{~Hz}$ and spatially filtered using xDAWN algorithm (Rivet et al., 2009). For each character and repetition the corresponding signal was defined as the sum of the one second EEG signal following its corresponding row intensification and the one following its corresponding column intensification. Trials were then classified using a Bayesian LDA classifier (MacKay, 1992). This type of classifier returns for each letter a probability that it belongs to the target class. Classification is calculated for each repetition, the final score for each character is computed as the sum of its probabilities for each repetition:

$$
s(l)=\sum_{i=1}^{n} p(l, i)
$$

where $s(l)$ is the score of the letter $l$ and $p(l, i)$ is the probability given to the letter $l$ by the LDA classifier at repetition $i$. The character to be spelled is chosen as the one having the highest final score. We will define this highest score $s_{1}$, the second highest score will be refered to as $s_{2}$ and so on. A ten-fold cross validation method was used.

\subsection{Results}

\subsubsection{Classification results}

In Figure 1 we plot the classification results for each subject versus the number of repetitions used. Without surprise the classification rate improves with the growing number of repetitions.

It is clear that one way of improving a P300 speller is simply to increase the number of repetitions, however this also increases the time spent to spell a letter and thus slows down the system.

\subsubsection{Second best score}

In a second time we got interested in the conditions of failure of our classifier. Thus we studied what happened when the highest score $\left(s_{1}\right)$ did not correspond

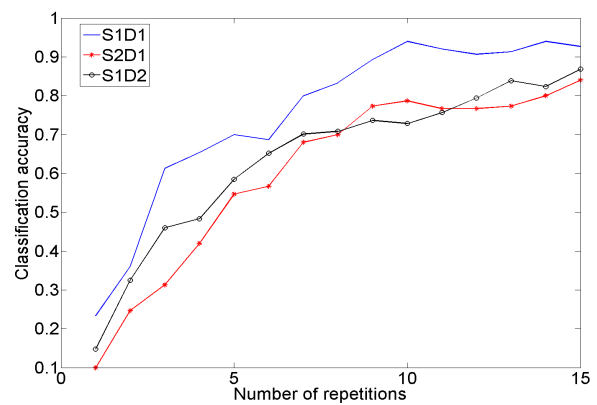

(a) Subject 1 (dataset 1)

Figure 1: Classification results for different subjects in function of the number of repetitions. Each line corresponds to a subject.

to the target character. Especially we looked at the probability that the second highest score $\left(s_{2}\right)$ corresponds to the target letter (when $s_{1}$ does not). In figure 2 we plot this probability for different subjects. We can see that this probability can be very low and very different from one subject to another and that no clear dependency on the number of repetitions can be deduced.

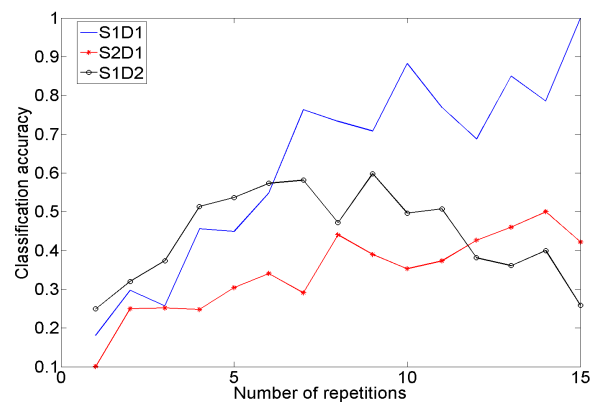

(a) Subject 1 (dataset 2)

Figure 2: Classification results using second highest score (when highest score is not the target) for different subjects. Each line corresponds to a subject.

\subsubsection{Highest score repartition}

Finally we studied the repartition of the highest score $\left(s_{1}\right)$ and the second highest score $\left(s_{2}\right)$. We separated our data into three classes:

- $s_{1}$ corresponds to the target letter.

- $s_{1}$ does not correspond to the target letter, $s_{2}$ does.

- neither $s_{1}$ nor $s_{2}$ correspond to the target letter.

Then we looked at the evolution of our scores $s_{1}$ and $s_{2}$ and of their difference $\left(s_{1}-s_{2}\right)$ in these different classes. In figure 3 we plot this repartition for the different subjects. In this figure we can see that for 
the three subjects $s_{1}$ is much lower when the target character corresponds to the second highest score $\left(s_{2}\right)$. The same observation can be made for their difference $\left(s_{1}-s_{2}\right)$ for which the effect is even stronger. This observation suggests that when $s_{1}-s_{2}$ is low the second highest score would correspond to the target character and inversely when $s_{1}-s_{2}$ is high the second highest score would not correspond to the target character.

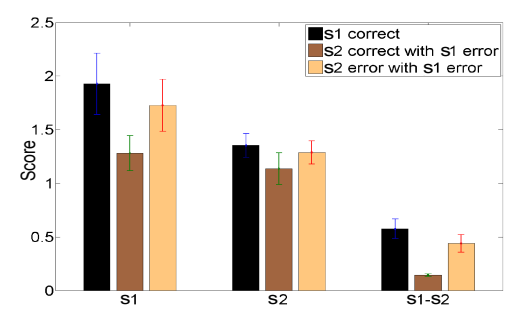

(a) Subject 1 (dataset 1)

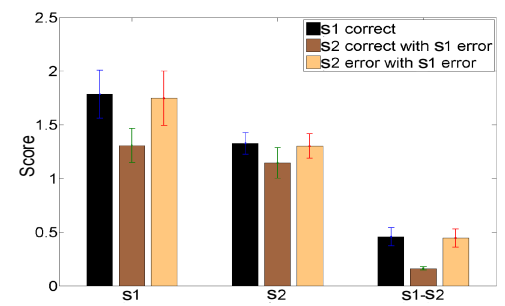

(b) Subject 2 (dataset 1)

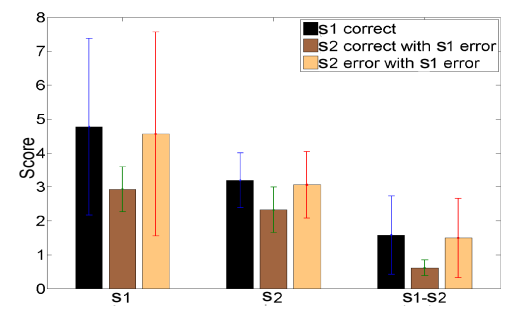

(c) Subject 1 (dataset 2)

Figure 3: Highest final scores after 10 repetitions.

We plot the mean value (standard error) for $s_{1}, s_{2}$ and their difference $\left(s_{1}-s_{2}\right)$ in three different conditions: 1$) s_{1}$ corresponds to the target letter, 2) $s_{2}$ corresponds to the target letter c)Neither $s_{1}$ nor $s_{2}$ correspond to the target letter.

\section{Propositions of integration}

Now that we have observed the performance of our P300 system and its characteristics we propose to simulate the integration of the ErrP in this system as an error correction system. As we have said before ErrP detection is not perfect and is subject to errors. Thus we will study the impact of our error-detection (ED) performances on our results.

\subsection{Integration}

ErrP can be integrated in different ways in BCI systems in order to correct errors. Using the previously presented observations we have selected four different strategies of integration:

- C: Canceling the command (i.e. erasing the letter). This method is the one that has been mostly used in previous studies on ErrP integration (Dal Seno et al., 2010).

- CI: Canceling the command and starting a new shorter session. The scores resulting from this new session will then be added to our original scores and a new decision will be made from our BCI system.

- R: Replacing the command by the one corresponding to the second highest score.

- RC: Replacing the command by the one corresponding to the second highest score only if the difference $s_{1}-s_{2}$ is lower than a predetermined threshold. If it is higher nothing is done.

\subsection{Performance measurement}

The performance of a BCI can be assessed in different ways depending on what we want to optimize. Here we used three different performance measures which evaluate different qualities of our system:

- Classification rate $\left(T_{f}\right)$ : this is the classification accuracy of our system for one trial. This is of great use if one desires a BCI that is very precise. The main disadvantage of this measure is that it does not take time into account. It is defined as:

$$
T_{f}=\frac{N b C}{N T o t}
$$

with $N b C$ being the number of trials correctly classified and NTot the total number of trials.

- Information transfer rate (ITR): this measure is a more complex one which takes into account both the classification accuracy, the time spent for a trial and the information it contains. With a classification accuracy of $T_{f}$, a trial duration of $c$ and $N$ possible outcomes, the ITR is defined as:

$B=\frac{\log _{2}(N)+T_{f} \log _{2}\left(T_{f}\right)+\left(1-T_{f}\right) \log _{2}\left(\frac{1-T_{f}}{N-1}\right)}{c}$

- Utility Metric (U): it was proposed by (Dal Seno et al., 2010). The main difference with the ITR is that here we consider the special case where the subject has to perform perfectly his task. Thus, when an error occurs the subject has to cancel the 
order and restart the trial. Let us define TL the time to perform correctly a task:

$$
T L=T_{f} c+\left(1-T_{f}\right)(c+T B+T L)=\frac{c}{2 T_{f}-1}
$$

where TB is the time spent to cancel the command, here we assume it is equivalent to any other command with $T B=T L$. The utility measure is then defined as:

$$
U=\frac{b L}{T L}
$$

where $b L$ is the benefit of a letter, if each letter is equiprobable then $b L=\log _{2}(N-1)$ bits.

\subsection{Threshold}

To determine the threshold for the RC strategy data were classified using the trained classifier, for each trial $i$ we have $D(i)=s_{1}(i)-s_{2}(i)$. D values are then divided into two groups: Group 1 contains trials for which $s_{1}$ corresponds to the target letter and Group 2 contains trials for which $s_{2}$ corresponds to the target letter. The threshold $\mathrm{T}$ is defined as the frontier between these two groups using LDA.

\subsection{Derivation of the performance measures}

Here we will present the different performance measures of the closed-loop system according to the chosen strategy.

\subsubsection{Local Variables}

Let us first define several variables:

- $T_{f}(n)$ : P300 classification rate with $\mathrm{n}$ repetitions.

- $T_{c}$ : ErrP classification rate for correct trials.

- $T_{e}$ : ErrP classification rate for error trials.

- $T_{f 2}$ : P300 classification rate using $s_{2}$ when $s_{1}$ is not correct.

- $T_{r 1}$ : Probability that $\left(s_{1}-s_{2}\right)<T$ when $s_{1}$ was not correct (i.e. error will be corrected).

- $T_{r 2}$ : Probability that $\left(s_{1}-s_{2}\right)>T$ when $s_{1}$ was correct (i.e. correct will not be modified).

- $T_{f c}: \mathrm{P} 300$ classification rate with $\mathrm{n}+\mathrm{p}$ repetitions when $T_{f}(n)$ was correct.

- $T_{f e}: \mathrm{P} 300$ classification rate with $\mathrm{n}+\mathrm{p}$ repetitions when $T_{f}(n)$ was not correct.

\subsubsection{Canceling strategy $(C)$}

Classification accuracy and ITR are defined as (refer to (Ferrez and Millán, 2005) for more details):

$$
\begin{gathered}
T_{f b}=\frac{T_{f} T_{c}}{T_{f} T_{c}+\left(1-T_{e}\right)\left(1-T_{f}\right)} \\
\operatorname{ITR}_{b}=s_{t} \frac{\log _{2}(N)+T_{f b} \log _{2}\left(T_{f b}\right)+\left(1-T_{f b}\right) \log _{2}\left(\frac{1-T_{f b}}{N-1}\right)}{c} \\
\text { with } s_{t}=\left(T_{f} T_{c}+\left(1-T_{e}\right)\left(1-T_{f}\right)\right) .
\end{gathered}
$$

The time spent to correctly spell a letter is (refer to (Dal Seno et al., 2010) for more details):

$$
T L=\frac{c}{T_{f} T_{c}+\left(1-T_{f}\right) T_{e}+T_{f}-1}
$$

\subsubsection{Canceling and iteration strategy (CI)}

Here trials detected as errors will be restarted and will have a new probability of being corrects giving a classification accuracy of:

$$
T_{f b}=T_{f} T_{c}+\left(1-T_{f}\right) T_{e} T_{f e}+T_{f}\left(1-T_{c}\right) T_{f c}
$$

Thus TL will be defined as:

$T L=\frac{\left(1+a\left(r_{p}\left(1-r_{c}\right)+r_{e}\left(1-r_{p}\right)\right)\right) c}{\left(1-2\left(r_{p}\left(1-r_{c}\right)\left(1-r_{p n 1}\right)+\left(1-r_{p}\right)\left(1-r_{e} r_{p n 2}\right)\right)\right.}$

Now we will only present $T_{f b}$, ITR and $\mathrm{U}$ can be derived by replacing $T_{f}$ by $T_{f b}$ in their definition.

\subsubsection{Replacement strategy $(R)$}

Here trials detected as errors are replaced by another letter thus we get for the classification accuracy:

$$
T_{f b}=T_{f} T_{c}+\left(1-T_{f}\right) T_{e} T_{f 2}
$$

\subsubsection{Replacement under condition strategy (RC)}

Here the difference with the previous method is that only a fraction of erroneously detected correct trials will be modified and only a proportion of correctly detected error trials will be corrected:

$$
T_{f b}=T_{f} T_{c}+T_{f}\left(1-T_{c}\right) T_{r 2}+\left(1-T_{f}\right) T_{e} T_{f 2} T_{r 1}
$$

\section{Results}

Integration was tested for $n \in[1,15]$ ( $\mathrm{n}$ is the number of repetitions). We used three new repetitions $(a=3)$ for the iteration strategy $(C I)$. Different performances of our error-detection system 


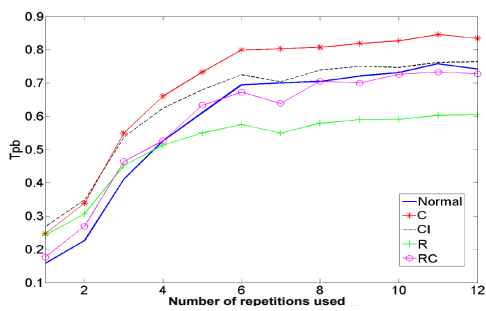

(a) $\mathrm{Tf}, \mathrm{Te}=0.6, \mathrm{Tc}=0.7$

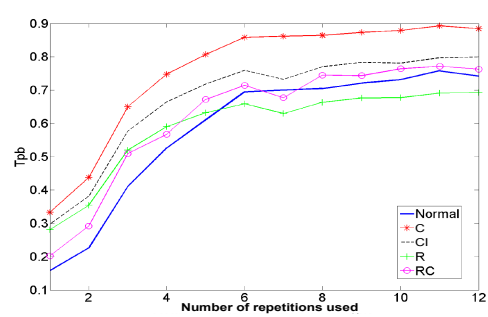

(d) Tf, Te=0.7, Tc=0.8

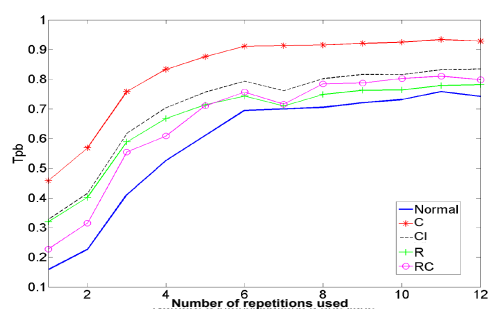

(g) Tf, Te=0.8, Tc=0.9

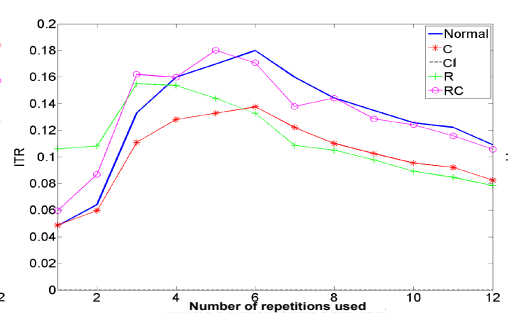

(b) ITR, Te=0.6, Tc=0.7

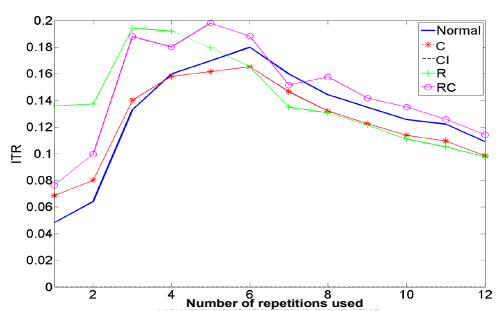

(e) ITR, Te=0.7, Tc $=0.8$

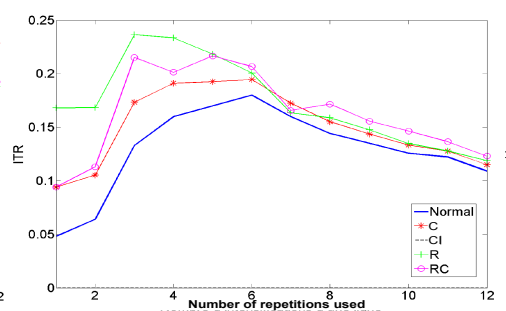

(h) ITR, Te $=0.8, \mathrm{Tc}=0.9$

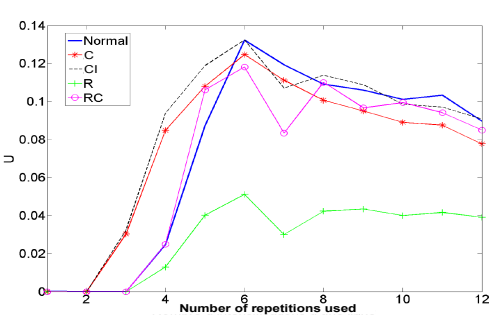

(c) $\mathrm{U}, \mathrm{Te}=0.6, \mathrm{Tc}=0.7$

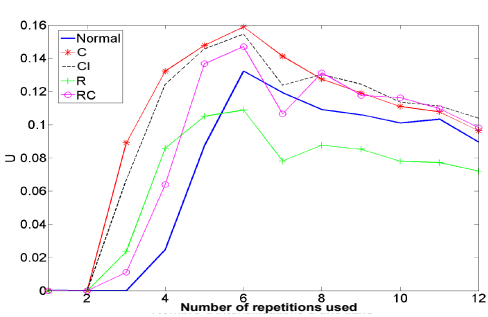

(f) $\mathrm{U}, \mathrm{Te}=0.7, \mathrm{Tc}=0.8$

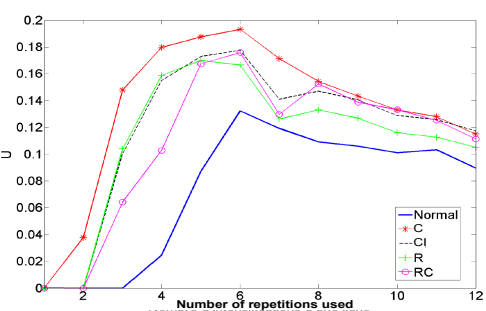

(i) $\mathrm{U}, \mathrm{Te}=0.8, \mathrm{Tc}=0.9$

Figure 4: Evolution of our different performance measures (Tf, ITR and $U$ ) with the number of repetitions used for different performances of our ED.Each column of plots correspond to a different measure of performance. Each line of plots correspond to a different performance of our ED. In each plot, each line corresponds to a different strategy of integration.

(ED) were tested: $T_{e} \in\{60 \%, 70 \%, 80 \%\}$ and $T_{c} \in$ $\{70 \%, 80 \%, 90 \%\}$. In figure 4 we plot the results for one subject. First of all one can see how much our performance measures are different. Each strategy performs differently for each performance measure. Here we present figures for only one subject but the observations made also apply to the other subjects.

\subsection{Classification accuracy}

Concerning the classification accuracy, one can see that this measure improves with growing number of repetitions for any type of integration strategy. Moreover, it seems that strategies $C$ and $C I$ allow improvement of the BCI system even for low performances of our $\mathrm{ED}\left(T_{e}=0.6\right.$ and $\left.T_{c}=0.7\right)$, with strategy $C$ being the most performant one. Strategies $R$ and $R C$ allow improvement of the initial system only for high performances of our ED $\left(T_{e} \geq 0.7\right.$ and $\left.T_{c} \geq 0.8\right)$ and never outperform strategy $C$. Thus it seems that if one aims at simply improving the classification accuracy of a system the best strategy of integration would be $C$ (ie, cancellation of a trial).

\subsection{Information transfer rate}

One can see that it does not increase with the growing number of repetitions, because the duration of a trial is taken into account. Integration results are very different from those obtained with $T_{f}$. Here strategy $C$ allows improvement only for high performance of our ED $\left(T_{e} \geq 0.8\right.$ and $\left.T_{c} \geq 0.9\right)$. Strategies $R$ and $R C$ are the best ones with strategy $R C$ being more stable than $R$ (for low ED performances $R C$ does not degrade our system while $R$ does). Finally one can note that the ITR follows the same evolution (for growing number of repetitions) before and after integration of our error correction system. If one aims at improving the ITR it seems that the best strategy would be $R C$ (ie, replacement under conditions). 


\subsection{Utility metric}

Once again, the utility metric (U) does not necessarily increase with growing number of repetitions. For low performances of our $\operatorname{ED}\left(T_{e}=0.6\right.$ and $\left.T_{c}=0.7\right)$ there does not seem to be one particular strategy clearly outperforming the others. However one can see that strategy $R$ gives very poor results. For higher performances of our $\operatorname{ED}\left(T_{e} \geq 0.8\right.$ and $\left.T_{c} \geq 0.9\right)$ it seems that strategy $A$ is slightly better than the other but this is not significant. However one can note that with these performances each strategy brings high improvement.

\subsection{Discussion}

In this paper we have simulated the integration of the ErrP as an error-correction system in a P300 speller BCI . We have presented different strategies of integration and studied their impact on the BCI performances. Using different types of performance measures we have seen that each strategy had some advantages and some disadvantages. The overall best strategy seems to be the strategy of canceling the erroneous order when it is detected since it gives the best results in terms of classification accuracy and of utility measure. However the measure used in most studies to assess the performance of multiclass BCIs is the ITR. For the ITR it seems that the best strategy is the strategy of replacing the erroneous command by the one obtaining the second best score under condition. Thus, one can not state for a best strategy. However what can be noted is that for high performances of our error-detection system every strategy allows improvement of our system for any type of performance measure. Moreover for $T_{e}=0.8$ and $T_{c}=0.9$ we get a mean improvement (over subject) of $11 \%$ of the classification accuracy, of $9 \%$ of the ITR and of $10 \%$ of the utility metric (each time for the best integration strategy). Thus we have seen that the ErrP could be used as an error-correction system in a multiclass BCI and that, even if it did not bring results as performant as for a two class BCI, one can get an improvement of around $10 \%$ of our system which is very encouraging. However we have also seen that the integration strategy should be carefully chosen according to the systems characteristics and to what one wants to improve.

\section{ACKNOWLEDGEMENTS}

We are grateful to the project ANR OpenVibe.

\section{REFERENCES}

Blankertz, B., Muller, K., Curio, G., Vaughan, T., Schalk, G., Wolpaw, J., Schlogl, A., Neuper, C., Pfurtscheller, G., Hinterberger, T., et al. (2004). The bci competition 2003: progress and perspectives in detection and discrimination of eeg single trials. Biomedical Engineering, IEEE Transactions on, 51(6):1044-1051.

Bollon, J., Chavarriaga, R., Millán, J., and Bessiere, P. (2009). EEG error-related potentials detection with a Bayesian filter.

Chavarriaga, R., Ferrez, P. W., and Millán, J. (2007). To err is human: Learning from error potentials in braincomputer interfaces. In R.Wang, Gu, F., and Shen, E., editors, Int Conf Cognitive Neurodynamics, pages 777-782, Shanghai, China.

Dal Seno, B., Matteucci, M., and Mainardi, L. (2010). The utility metric: a novel method to assess the overall performance of discrete brain-computer interfaces. Neural Systems and Rehabilitation Engineering, IEEE Transactions on, 18(1):20-28.

Falkenstein, M., Hohnsbein, J., Hoormann, J., and Blanke, L. (1991). Effects of crossmodal divided attention on late ERP components.II. Error processing in choice reaction tasks. Electroencephalogr. Clin. Neurophysiol., 78:447-455.

Farwell, L. A. and Donchin, E. (1988). Talking off the top of your head: toward a mental prosthesis utilizing event-related brain potentials. Electroencephalogr. Clin. Neurophysiol., 70(6):510-523.

Ferrez, P. and Millán, J. (2005). You are wrong!—automatic detection of interaction errors from brain waves. In Proceedings of the 19th International Joint Conference on Artificial Intelligence, 2005.

Ferrez, P. and Millán, J. (2007). EEG-based brain-computer interaction: Improved accuracy by automatic singletrial error detection. In Proc. NIPS 20.

Ferrez, P. and Millán, J. (2008). Simultaneous real-time detection of motor imagery and error-related potentials for improved BCI accuracy. In Proc 4th Intl. BrainComputer Interface Workshop and Training Course, Graz, Austria.

Gehring, W., Goss, B., Coles, M., Meyer, D., and Donchin, E. (1993). A neural system for error detection and compensation. Psychol. Sci., 4(Suppl 6):385-390.

Gentsch, A., Ullsperger, P., and Ullsperger, M. (2009). Dissociable medial frontal negativities from a common monitoring system for self-and externally caused failure of goal achievement. Neuroimage, 47(4):20232030.

MacKay, D. (1992). Bayesian interpolation. Neural computation, 4(3):415-447.

Rivet, B., Souloumiac, A., Attina, V., and Gibert, G. (2009). xDAWN algorithm to enhance evoked potentials: Application to brain-computer interface. Biomedical Engineering, IEEE Transactions on, 56(8):2035-2043.

Steinhauser, M. and Kiesel, A. (2011). Performance monitoring and the causal attribution of errors. Cognitive, Affective, \& Behavioral Neuroscience, pages 1-12. 